\title{
Self-Care-Based Treatment Using Ordinary Elastic Bandages for Venous Leg Ulcers
}

\author{
Kotaro Suehiro, MD, Noriyasu Morikage, MD, Takasuke Harada, MD, Makoto Samura, MD, \\ Yuriko Takeuchi, MD, Takahiro Mizoguchi, MD, and Kimikazu Hamano, MD
}

\begin{abstract}
Objective: We aimed to study venous leg ulcer (VLU) healing and recurrence rates of VLU using a self-care-based treatment strategy.

Methods: The study included 36 patients (43 legs) who visited our clinic between April 2009 and June 2015 because of non-healing VLUs and who had been treated by us for more than a year (until June 2016). Patients or their caregivers were first provided instructions for performing the "no-intentional-stretch" bandaging technique using ordinary elastic bandages. Wounds were cleansed with tepid water daily, and bandages were re-applied by patients or their caregivers; this was continued until VLUs were healed. Compression was discontinued after healing, but was restarted if persistent swelling and/or dermatitis was noticed on their legs.
\end{abstract}

Results: The median ulcer size was $6.5 \mathrm{~cm}^{2}$ (range, $1-105 \mathrm{~cm}^{2}$ ). The median number of clinic visits until healing was six (range, 3-35). The 6- and 12-month healing rates were $67 \%$ and $86 \%$, respectively. Twenty (44\%) legs required compression therapy after VLU healing. The cumulative recurrence-free rate at 60 months was $86 \%$.

Conclusion: Reasonable healing and recurrence rates were achieved by applying a self-care-based VLU treatment strategy.

Keywords: venous leg ulcer, compression therapy, elastic bandage

\section{Introduction}

Venous leg ulcer (VLU) is one of the most common problems for which patients seek medical care. ${ }^{1)}$ Since the time of Hippocrates, compression therapy has been considered as the gold standard for VLU treatment. ${ }^{2)}$ Majority of

Division of Vascular Surgery, Department of Surgery and Clinical Science, Yamaguchi University Graduate School of Medicine, Ube, Yamaguchi, Japan

Received: March 28, 2017; Accepted: July 11, 2017

Corresponding author: Kotaro Suehiro, MD. Division of Vascular Surgery, Department of Surgery and Clinical Science, Yamaguchi University Graduate School of Medicine, 1-1-1 Minamikogushi, Ube, Yamaguchi 755-8505, Japan

Tel: +81-836-22-2260, Fax: +81-836-22-2423

E-mail: ksuehiro-circ@umin.ac.jp
VLUs get healed as long as proper compression is applied, regardless of modalities. ${ }^{3)}$ Similarly, non-compliance with compression therapy is known to lower healing rates and increase recurrence rates. ${ }^{4)}$ However, there is a shortage of expert phlebologists and nurses in Japan. In addition, compression hosieries and bandages for chronic venous diseases are not covered by national insurance policies, which results in the discontinuation of medical care in VLU patients. In this study, we primarily treated VLU patients with a self-care-based strategy involving "nointentional-stretch" bandaging technique and evaluated the effectiveness of our strategy to reduce patient burdens and to standardize the quality of compression therapy.

\section{Methods}

This retrospective study was approved by our Institutional Review Board, and the need for obtaining individual patient consent was waived. Between April 2009 and June 2015, 41 patients visited our clinic because of non-healing VLUs (VLUs that were not healed for $>6$ months); of these patients, 36 (43 legs) were treated by us for more than a year (until June 2016) and were included in the study. Patient characteristics are summarized in Table 1. Duplex ultrasonography (DUS) was performed in all patients. However, because venous ulcers can develop without showing any abnormality in DUS, ${ }^{5)}$ we regarded ulcers in the area of lipodermatosclerosis as those with a venous origin. In difficult cases, the diagnosis was made based on mutual agreement between us, dermatologists, and pathologists.

\section{Management of VLUs}

In the early stages of VLU treatment, surgical interventions for superficial and/or perforator vein insufficiency were performed, if indicated. Deep vein surgery was not performed because our clinic did not have an expert surgeon. For compression therapy, we instructed patients to apply bandages between the ankle and popliteus and to use three rolls of ordinary elastic bandages $\left(\right.$ Getto $^{\circledR}$ elastic bandage, Kawamoto Corporation, Osaka, Japan; extensibility, $108 \%$; width, $10 \mathrm{~cm}$; length, $4.5 \mathrm{~m} /$ roll; cost, 450 
Table 1 Characteristics of patients, affected legs, and associated ulcers

\begin{tabular}{|c|c|c|}
\hline Patients & & $(N=36)$ \\
\hline \multirow[t]{2}{*}{ Age } & Years; median (range) & $68(28-91)$ \\
\hline & $>70$ years & $17(47 \%)$ \\
\hline Sex & Male: female & $14: 22$ \\
\hline \multirow[t]{2}{*}{ BMI } & $\mathrm{kg} / \mathrm{m}^{2} ;$ median (range) & $23(16-42)$ \\
\hline & $>30 \mathrm{~kg} / \mathrm{m}^{2}$ & $7(19 \%)$ \\
\hline Walking distance & $<200 \mathrm{~m} /$ day & $7(19 \%)$ \\
\hline Occupation & Standing for $>8 \mathrm{~h} /$ day & $9(25 \%)$ \\
\hline Rheumatoid arthritis & & $7(19 \%)$ \\
\hline \multirow{2}{*}{$\begin{array}{l}\text { Previous admission } \\
\text { for VLUs }\end{array}$} & & $9(25 \%)$ \\
\hline & Multiple admissions & $4(11 \%)$ \\
\hline Legs & & $(N=43)$ \\
\hline AROM & $<20^{\circ}$ & $17(40 \%)$ \\
\hline \multirow[t]{5}{*}{ Venous pathology } & FV reflux & $1(2 \%)$ \\
\hline & FV occlusion & $0(0 \%)$ \\
\hline & GSV reflux & $15(35 \%)$ \\
\hline & SSV reflux & $2(5 \%)$ \\
\hline & PV reflux & $1(2 \%)$ \\
\hline \multirow[t]{2}{*}{ Previous surgery } & $\begin{array}{l}\text { Varicose, perforator } \\
\text { veins }\end{array}$ & $3(7 \%)$ \\
\hline & Skin grafting & $7(16 \%)$ \\
\hline \multicolumn{3}{|l|}{ Ulcers } \\
\hline $\begin{array}{l}\text { Duration before } \\
\text { treatment }\end{array}$ & $\begin{array}{l}\text { Years; } \\
\text { median (range) }\end{array}$ & $0.6(0.5-12)$ \\
\hline \multirow[t]{2}{*}{ Laterality } & Right: left & $22: 21$ \\
\hline & Bilateral & $7(16 \%)$ \\
\hline \multirow[t]{2}{*}{ Recurrences } & & $15(35 \%)$ \\
\hline & $\begin{array}{l}\text { Average number of } \\
\text { recurrences }\end{array}$ & 0.8 \\
\hline Size & $\mathrm{cm}^{2} ;$ median (range) & $6.5(1-105)$ \\
\hline \multirow[t]{6}{*}{ Location } & Lower inner calf & $24(56 \%)$ \\
\hline & Lower lateral calf & $20(47 \%)$ \\
\hline & Inner ankle & $9(21 \%)$ \\
\hline & Lateral ankle & $0(0 \%)$ \\
\hline & Pedal & $2(5 \%)$ \\
\hline & Multiple & $13(30 \%)$ \\
\hline
\end{tabular}

BMI: body mass index; AROM: ankle range of motion; FV: femoral vein; GSV: great saphenous vein; SSV: short saphenous vein; $\mathrm{PV}$ : perforator vein

JPY/roll), which can be repeatedly washed and reused for several months. Details of the application technique have been reported previously. ${ }^{6}$ Briefly, the person applying the bandage was instructed to softly place it around the leg so that it can fit the leg and smooth out wrinkles in an advancing direction without intentionally stretching the bandage. This technique effectively reduced variations in interface pressure (IP) and stiffness introduced depending on the skills of the person applying the bandage and also standardized the compression level among patients. Further, elastic bandages provided a better fit with minimal tension compared with inelastic bandages. With this technique, IP and stiffness linearly increased with the number of bandage rolls used. Using three rolls of bandages between the ankle and popliteus, an IP of $40-45 \mathrm{mmHg}$ and static stiffness index (SSI) ${ }^{7}$ of $11-13 \mathrm{mmHg}$ can be achieved in ordinary Japanese patients. ${ }^{8)}$ In cases where adequate IP and/or stiffness could not be achieved using three rolls to promote healing, additional rolls were added or the outermost bandage was replaced with an inelastic one. In most cases, another roll was required for the pedal to control edema. Bandages were applied for $24 \mathrm{~h}$ a day, except during bathing, until VLUs were healed. Wounds were cleansed using tepid water, and bandages were reapplied daily either by patients or by their caregivers. Medications such as iodine/sugar paste and basic fibroblast growth factor (Fiblast ${ }^{\circledR}$, Kaken Pharmaceutical Co., Ltd., Tokyo, Japan) were used, if indicated. Wounds were covered using simple non-adherent dressings. In cases where exudates were abundant, non-sterile diapers were directly applied. Initially, patients or caregivers were asked to visit the clinic every 1-2 weeks until they mastered the bandaging technique for appropriate wound management. Once they had perfected the technique, they visited the clinic once every 1-3 months, and we examined the wounds and bandaging techniques. During every visit, two measurements of the maximal perpendicular diameter of VLU were recorded, and these values were multiplied to determine the ulcer size as described by Stacey et al. ${ }^{91}$ VLU was considered to be healed when epithelialization of the entire wound surface was achieved. After healing, we encouraged patients to discontinue compression therapy. Compression therapy with bandages or use of strong medical compression stockings (MCSs) was restarted only if persistent swelling and/or dermatitis was noticed on their legs.

\section{Statistical analysis}

Results are presented as mean \pm standard deviation or count (percentage) unless otherwise indicated. KaplanMeier analysis was performed to estimate the recurrencefree survival. Statistical analyses were performed using JMP 11.0 (SAS Institute, Cary, NC, USA). A p-value of $<0.05$ was considered statistically significant.

\section{Results}

The median number of clinic visits to learn the bandage technique was two (range, 1-7) during a median of two (range, 1-8) weeks. Seven patients had physical disabilities, and bandages were applied by their caregivers, and the remaining $29(81 \%)$ patients themselves applied bandages. Two patients applied bandages for $<3$ days per week, but the remaining 34 (94\%) patients complied with 


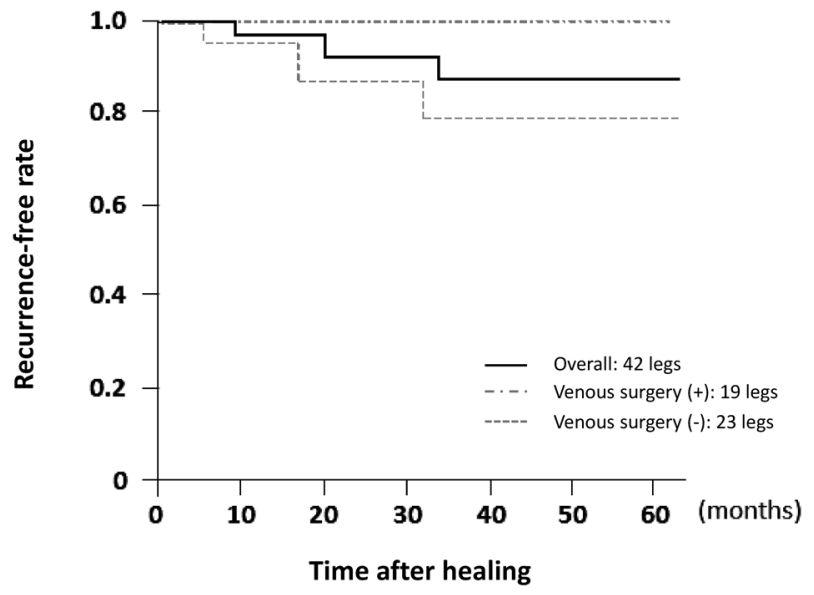

Fig. 1 Recurrence-free rate.

the instruction, i.e., bandages were applied for $24 \mathrm{~h}$ a day, except during bathing. The median number of visits to the clinic until healing was six (range, 3-35) during a median of five (range, 0.6-62) months. In 42 (98\%) legs, VLUs were healed with the aforementioned method. In the case of an 83-year-old woman, despite treating a $4.8-\mathrm{cm}^{2} \mathrm{VLU}$ in the gaiter area for a year, the ulcer did not heal possibly because of severe anemia. This was finally managed using hyperbaric oxygen therapy. The 6- and 12-month healing rates were $67 \%$ and $86 \%$, respectively.

Among the 19 legs on which venous surgery was performed, compression therapy was restarted for four $(21 \%)$ legs after healing (two bandages and two MCSs). Of the 23 legs on which surgery was not indicated, compression therapy was restarted in 16 (70\%) after healing (11 bandages and five MCSs). In this group, VLUs recurred on five legs, which were successfully treated by applying the same self-care-based strategy. The cumulative recurrence-free rate at 60 months was $86 \%$ (Fig. 1). Patients that were indicated for venous surgery were recurrence-free during the current observation period, while the recurrence-free probability was $78 \%$ at 60 months in patients who were not indicated for surgery. Because the recurrence rate was low and the sample size was small, risk analysis for recurrence was not performed.

\section{Discussion}

A general recommendation of applying compression therapy for a prolonged period because of high recurrence rates of VLUs imposes significant economic and social burdens on patients. ${ }^{4,10)}$ In Western countries, medical personnel who provide compression therapy significantly contribute to treatment costs. ${ }^{11)}$ Elderly individuals are at an increased risk for developing VLUs ${ }^{12)}$; however, they also find it difficult to make frequent hospital visits. Our self-care-based strategy for the healing of VLUs at home may contribute to reducing these burdens while maintaining comparable healing and recurrence-free rates compared with those reported previously. ${ }^{3,4,13,14)}$ In fact, during the treatment, patients gained confidence in managing the VLU treatment by themselves and found it unnecessary to make frequent hospital visits, which altogether resulted in a reduced number of hospital visits.

VLUs are open wounds with an active blood supply and these should be spontaneously healed if venous stasis is properly managed. Therefore, the application of an appropriate compression level is critical for the healing of VLUs. Compared with custom-made hosieries that require regular refitting, ordinary elastic bandages are cheaper, reusable, and can fit legs of any shape, even when used with dressings. These bandages are particularly beneficial for patients in Japan where hosieries for venous diseases are not covered by national insurance policies. Moreover, patient compliance with the use of strong MCSs is known to be very poor. ${ }^{15,16)}$ Further, elderly individuals prefer bandages because they are easy to use. ${ }^{17)}$ Our "no-intentionalstretch" technique rarely led to complications, such as ischemia and skin injury due to constriction, and ensured that IP and stiffness could be easily maintained and controlled. ${ }^{8)}$ This bandaging technique is expected to generate an IP of $40-45 \mathrm{mmHg}$ and an SSI of $11-13 \mathrm{mmHg}$, and the values measured at the clinic were similar to these values. It has been reported that a higher IP is associated with a higher VLU healing rate. ${ }^{18)}$ Mosti et al. have recently reported that VLUs with moderate ischemia can be successfully managed with an IP of approximately $40 \mathrm{mmHg}{ }^{19)}$; however, an IP of approximately $50 \mathrm{mmHg}$ can reduce blood flow to the toe. ${ }^{20)}$ These results indicate that an IP of $40-50 \mathrm{mmHg}$, which is equivalent to the values observed in our study, may be sufficient and safe for the healing of VLUs. It has also been reported that the healing of VLUs is strongly related with a higher stiffness. ${ }^{21)}$ Importantly, the bandaging technique applied by Mosti et al. generated an SSI as high as $18 \mathrm{mmHg}$ even at the time of bandage removal. However, our results suggest that an SSI of $>10 \mathrm{mmHg}$ may be sufficient for the healing of most VLUs.

In the present study, the probability of VLU recurrence in patients who underwent superficial and/or perforator surgery was less, although some of them $(21 \%$ of legs) required some form of compression therapy after healing. Thomas et al. have reported that $52 \%$ of legs do not require compression therapy after the healing of VLUs by venous surgeries. ${ }^{22)}$ Therefore, it can be assumed that some patients with VLUs due to superficial and/or perforator vein insufficiency do not require lifelong compression therapy. However, despite the absence of venous disorders in a duplex scan, other risk factors, such as occupations requiring prolonged standing peri- 
ods, ${ }^{5)}$ obesity, and reduced ankle range of motion, ${ }^{23)}$ can lead to VLUs. VLU recurrence is always preceded by C3-4 symptoms described by the Clinical-Etiology-AnatomyPathophysiology classification. ${ }^{24)}$ Therefore, we recommend that patients should discontinue the compression therapy once the ulcers are healed and should restart the therapy only when symptoms of recurrence are noticed. Because patients had already mastered the self-care based strategy, they could easily restart it, if needed. We speculate that this strategy will relieve a considerable number of VLU patients from life-long compression therapy.

\section{Limitation}

Because this was a single-center and retrospective study that included a limited number of patients, reaching a definite conclusion was difficult. Although IP and SSI are important factors in compression therapy, methods such as Holter electrocardiography used to monitor these factors in real time are lacking; such methods need to be developed in the future.

\section{Conclusion}

Healing and recurrence-free rates obtained with our selfcare-based treatment strategy for VLU were comparable to those reported previously. We believe that this strategy has the potential to reduce economic and social burdens on patients.

\section{Acknowledgments}

None.

\section{Funding}

This research received no specific grant from any funding agency in the public, commercial, or not-for-profit sectors.

\section{Ethical Approval}

This retrospective study was approved by the Institutional Review Board of Yamaguchi University Hospital (Center for Clinical Research, Ube, Yamaguchi, Japan), and the need for obtaining individual patient consent was waived.

\section{Disclosure Statement}

The authors declare that there is no conflict of interest.

\section{Author Contributions}

Study conception: KS

Data collection: KS, TH, MS, YT, TM
Analysis: KS

Investigation: KS

Writing: KS

Funding acquisition: NM, KH

Critical review and revision: all authors

Final approval of the article: all authors

Accountability for all aspects of the work: all authors

\section{References}

1) Abbade LPF, Lastória S, de Almeida Rollo H. Venous ulcer: clinical characteristics and risk factors. Int J Dermatol 2011; 50: 405-11.

2) Raffetto JD. Venous ulcer formation and healing at cellular levels. In: Handbook of Venous Disorders: Guidelines of the American Venous Forum, 3rd edition. Oxofrd: Oxford University Press, 2009: 70-82.

3) Chi YW, Raffetto JD. Venous leg ulceration pathophysiology and evidence based treatment. Vasc Med 2015; 20: 168-81.

4) Mayberry JC, Moneta GL, Taylor LM Jr, et al. Fifteen-year results of ambulatory compression therapy for chronic venous ulcers. Surgery 1991; 109: 575-81.

5) Suehiro K, Morikage N, Yamashita O, et al. Risk factors in patients with venous stasis-related skin lesions without major abnormalities on duplex ultrasonography. Ann Vasc Dis 2016; 9: 201-4.

6) Suehiro K, Okada M, Yoshimura A, et al. Elastic multilayer bandages for chronic venous insufficiency: features of our technique. Ann Vasc Dis 2012; 5: 347-51.

7) Partsch H. The static stiffness index: a simple method to assess the elastic property of compression material in vivo. Dermatol Surg 2005; 31: 625-30.

8) Suehiro K, Morikage N, Murakami M, et al. Study on different bandages and application techniques for achieving stiffer compression. Phlebology 2015; 30: 92-7.

9) Stacey MC, Burnand KG, Layer GT, et al. Measurement of the healing of venous ulcers. Aust N Z J Surg 1991; 61: 8448.

10) Lal BK. Venous ulcers of the lower extremity: definition, epidemiology, and economic and social burdens. Semin Vasc Surg 2015; 28: 3-5.

11) Partsch H, Mortimer P. Compression for leg wounds. Br J Dermatol 2015; 173: 359-69.

12) Eberhardt RT, Raffetto JD. Chronic venous insufficiency. Circulation 2014; 130: 333-46.

13) Barwell JR, Davies CE, Deacon J, et al. Comparison of surgery and compression with compression alone in chronic venous ulceration (ESCHAR study): randomised controlled trial. Lancet 2004; 363: 1854-9.

14) Marston WA, Carlin RE, Passman MA, et al. Healing rates and cost efficacy of outpatient compression treatment for leg ulcers associated with venous insufficiency. J Vasc Surg 1999; 30: 491-8.

15) Raju S, Hollis K, Neglen P. Use of compression stockings in chronic venous disease: patient compliance and efficacy. Ann Vasc Surg 2007; 21: 790-5.

16) Nelson EA, Harper DR, Prescott RJ, et al. Prevention of recurrence of venous ulceration: randomized controlled trial of class 2 and class 3 elastic compression. J Vasc Surg 2006; 
44: 803-8.

17) Suehiro K, Morikage N, Yamashita O, et al. Adherence to and efficacy of different compression methods for treating chronic venous insufficiency in the elderly. Phlebology 2016; 31: 723-8.

18) O’Meara S, Cullum NA, Nelson EA. Compression for venous leg ulcers. Cochrane Database Syst Rev 2009; CD000265.

19) Mosti G, Cavezzi A, Massimetti G, et al. Recalcitrant venous leg ulcers may heal by outpatient treatment of venous disease even in the presence of concomitant arterial occlusive disease. Eur J Vasc Endovasc Surg 2016; 52: 385-91.

20) Mosti G, Iabichella ML, Partsch H. Compression therapy in mixed ulcers increases venous output and arterial perfusion. J Vasc Surg 2012; 55: 122-8.
21) Milic DJ, Zivic SS, Bogdanovic DC, et al. The influence of different sub-bandage pressure values on venous leg ulcers healing when treated with compression therapy. J Vasc Surg 2010; 51: 655-61.

22) Thomas CA, Holdstock JM, Harrison CC, et al. Healing rates following venous surgery for chronic venous leg ulcers in an independent specialist vein unit. Phlebology 2013; 28: 132-9.

23) Verma H, Tripathi RK. Algorithm-based approach to management of venous leg ulceration. Semin Vasc Surg 2015; 28: 54-60.

24) Porter JM, Moneta GL; International Consensus Committee on Chronic Venous Disease. Reporting standards in venous disease: an update. J Vasc Surg 1995; 21: 635-45. 